\title{
Peroral cholangioscopy by SpyGlass DS versus CHF-B260 for evaluation of the lateral spread of extrahepatic cholangiocarcinoma
}

(1) $\odot \ominus$

\begin{abstract}
Authors

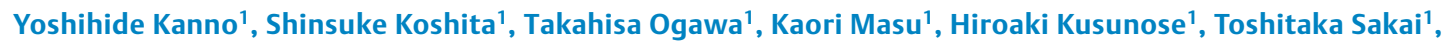
Toji Murabayashi ${ }^{1}$, Sho Haegawa', Fumisato Kozakai ${ }^{1}$, Keisuke Yonamine ${ }^{1}$, Yujiro Kawakami ${ }^{1}$, Yuki Fujii ${ }^{1}$, Jun Horaguchi ${ }^{1,2}$, Yutaka Noda ${ }^{1}$, Kei Ito ${ }^{1}$
\end{abstract}

Institutions

1 Sendai City Medical Center - Gastroenterology, Sendai, Japan

2 Natori Chuo Clinic - Gastroenterology, Natori, Japan

submitted 8.3.2018

accepted after revision 24.7.2018

Bibliography

DOI https://doi.org/10.1055/a-0743-5283 |

Endoscopy International Open 2018; 06: E1349-E1354

(c) Georg Thieme Verlag KG Stuttgart · New York

ISSN 2364-3722

Corresponding author

Yoshihide Kanno, Sendai City Medical Center -

Gastroenterology, 5-22-1 Tsurugaya Miyagino-ku, Sendai

983-0824, Japan

Fax: +81-22-252-9431

yoshi-hk@openhp.or.jp

\section{ABSTRACT}

Background and study aims A newly developed peroral cholangioscopy (POCS) system, SpyGlassDS has high maneuverability. This study aimed to evaluate acceptability of the accuracy of SpyGlassDS accompanied by simultaneous POCS-guided biopsy compared with that of a traditional POCS scope, CHF-B260, to diagnose the lateral extent of extrahepatic cholangiocarcinoma (LEC).
Patients and methods Patients who underwent surgical resection after preoperative examinations to diagnose LEC were evaluated. POCS by CHF-B260 was performed if there was discrepancy between preceding fluoroscopy-guided biopsy findings and other examinations between January 2004 and September 2015 (group A, n=56); and POCS plus POCS-guided mapping biopsy by SpyGlassDS was performed in all surgical candidates between October 2015 and December 2017 (group B, n=20). The main outcome measure was the accuracy of overall preoperative diagnosis (OPD) of LEC defined based on all examinations, including POCS.

Results Accuracy of OPD for the liver side and the ampullary side was $93 \%$ and $100 \%$, respectively, in group A, and $84 \%$ and $100 \%$, respectively, in group B ( $P=0.37$ for the liver side; $P$, not available for the ampullary side). Diagnostic accuracy of simple optical evaluation by POCS for the liver side and the ampullary side was $83 \%$ and $100 \%$, respectively, in group $\mathrm{A}$, and $58 \%$ and $88 \%$, respectively, in group $\mathrm{B}(P=0.29$ for the liver side; $P=0.40$ for the ampullary side). Conclusions POCS by SpyGlassDS was found to be acceptable and could be a standard approach for diagnosis of LEC.

\section{Clinical.Trials.gov}

UMIN000030583

TRIAL REGISTRATION: Single-center, non-randomized, retrospective study

UMIN000030583 at clinicaltrials.gov

\section{Introduction}

The sole possible curative treatment for extrahepatic bile duct cancer is surgical resection [1-3]. Unresectability of this cancer is associated with poor prognosis. However, lateral spreading of this cancer along the longitudinal axis of the bile duct often results in non-curative resection because of detection difficulties encountered in imaging examinations [4,5]. Diagnosing the precise borderlines of the disease is key to achieve curative resection.

Of the two types of lateral spread, intraepithelial spread is extremely difficult to diagnose with cross-sectional images, whereas intramural spread is relatively easy to identify on contrast-enhanced computed tomography (CT). Although intraepithelial spread can be diagnosed with endoscopic ultrasound (EUS) or intraductal ultrasound (IDUS) $[6,7]$ in most cases, it is 
undetectable when the neoplasm only causes low epithelial changes. Cholangioscopy can possibly result in a proper diagnosis for difficult intraepithelial spread [8-10]. After several brilliant studies confirmed the value of both percutaneous transhepatic cholangioscopy and peroral duodenoscope-assisted cholangioscopy (POCS) with fiberscopes [11-15], digital scopes with higher imaging quality became available. For instance, a CHF-B260 scope from Olympus Corporation (Tokyo, Japan), which has the highest level of imaging quality among existing cholangioscopes, has been available and is reported to have higher capability to accurately diagnose such lateral extent $[10,16]$.

Recently, the SpyGlass DS system from Boston Scientific Corporation (Marlborough, Massachusetts, United States) has become commercially available. This system consists of a disposable digital scope with a field of view of 120 degrees. Although the spatial quality of the system is considered to be relatively low in comparison with that of the Olympus scope, the scope has a tapered tip, a four-way tip deflection system, and a dedicated channel for water irrigation, which allow unimpeded observation and biopsy procedures [17-21]. There have been no reports comparing these two types of digital cholangioscopes. Therefore, we conducted such a comparison in a retrospective design and reported the results herein.

\section{Patients and methods}

\section{Patients}

All data on patients who underwent surgical resection for extrahepatic bile duct cancer after preoperative examinations, including POCS, to diagnose the lateral extent of extrahepatic cholangiocarcinoma at Sendai City Medical Center were extracted from a prospectively maintained database of endoscopic retrograde cholangiopancreatography. The following patients were excluded: (1) those in whom the clinical record on findings of the POCS examination could not be obtained or was insufficient for evaluation; (2) those who did not undergo surgical resection after examinations; and (3) those in whom the resected specimen was inappropriate to precisely evaluate the lateral extent. Patients who underwent examinations between January 2004 and September 2015 were included in the POCS by CHFB260 (CHF) group (group A) and those who underwent examinations since the SpyGlass DS system was employed in October 2015 were included in the SpyGlass DS group (group B).

\section{Procedures}

Mapping biopsy under $\mathrm{x}$-ray fluoroscopy (endoscopic retrograde cholangiography [ERC]) without POCS was performed in all patients in group A ( $>$ Fig. $\mathbf{1}$ ). In this group, POCS was performed when diagnosis of tumor spread by mapping biopsy findings was questionable because of possible discrepancy between ERC-guided mapping biopsy findings and other examinations, such as ERC, IDUS, EUS, CT, and magnetic resonance imaging (MRI) ( $\triangleright$ Fig. 2). This group included patients who did not undergo POCS because of absence of such discrepancy. Radial jaw biopsy forceps (Boston Scientific) were used for ERCguided mapping biopsy.

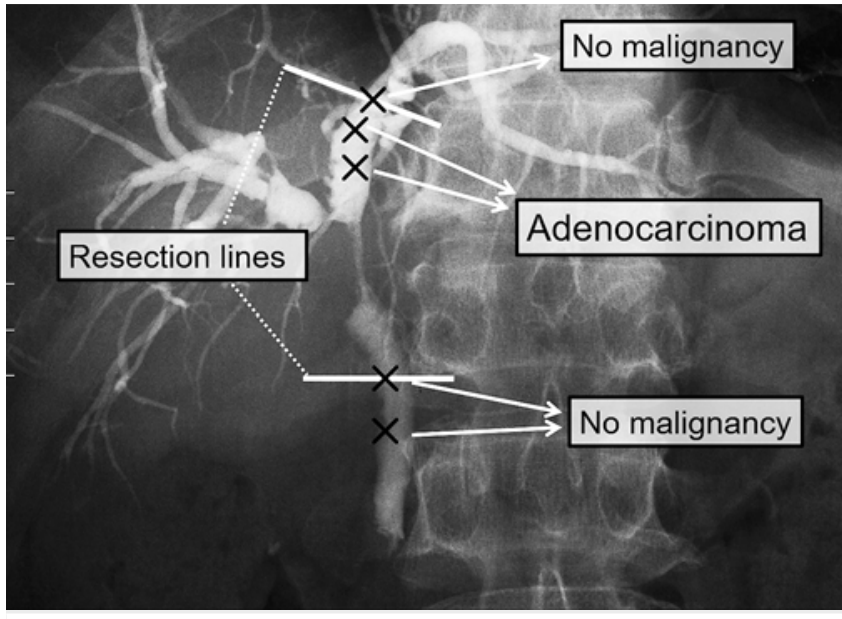

- Fig. 1 Endoscopic cholangiography-guided mapping biopsy. When the extrahepatic cholangiocarcinoma is obviously and largely involved the right hepatic duct, the border lines in the left hepatic duct and the lower bile duct should be identified. If the mapping biopsy revealed that the cancer extent did not reach the B3/B4 bifurcation and the level of the upper edge of the pancreas, this cholangiocarcinoma would be curatively removed by resection at the shown lines.

In group B, POCS using SpyGlass DS was performed without reference of ERC-guided mapping biopsy. The SpyGlass DS system was considered to be important as a biopsy instrument with visual confirmation of the target sites rather than a diagnostic device for obtaining endoscopic optical findings. SpyBite biopsy forceps (Boston Scientific) were used for POCS-guided biopsy in this group.

Both types of scopes were managed in the mother-baby style, that is, handled through a duodenoscope (TJF260V, Olympus). Endoscopic sphincterotomy was performed in the same or previous session in all patients. When the POCS procedure, which required injection of a large amount of water into the bile duct, was considered to be inappropriate in the first session because of accompanying acute cholangitis, it was performed in the next session after drainage with a plastic stent. After insertion of the cholangioscope with or without guidewire assistance, turbid bile and contrast were replaced by saline injected through the working channel of the CHF-B260 scope or through the irrigation channel of the SpyGlass DS scope. A guidewire or contrast solution was used to confirm the anatomical branch, if necessary. When it was difficult to insert the cholangioscope through the stricture, a bougie dilator or a 10Fr plastic stent was used for dilation. Endoscopic optical findings obtained from POCS images, including the location of the cancer borderlines, were reported in detail by the examiner.

\section{Outcome measurements and definitions}

The main outcome measurement was defined as accuracy of the overall preoperative diagnosis of tumor lateral extent by the biopsy results and POCS findings in each group. Because there would be selection bias due to the different diagnostic strategies in each group, diagnostic accuracy of pure POCS 

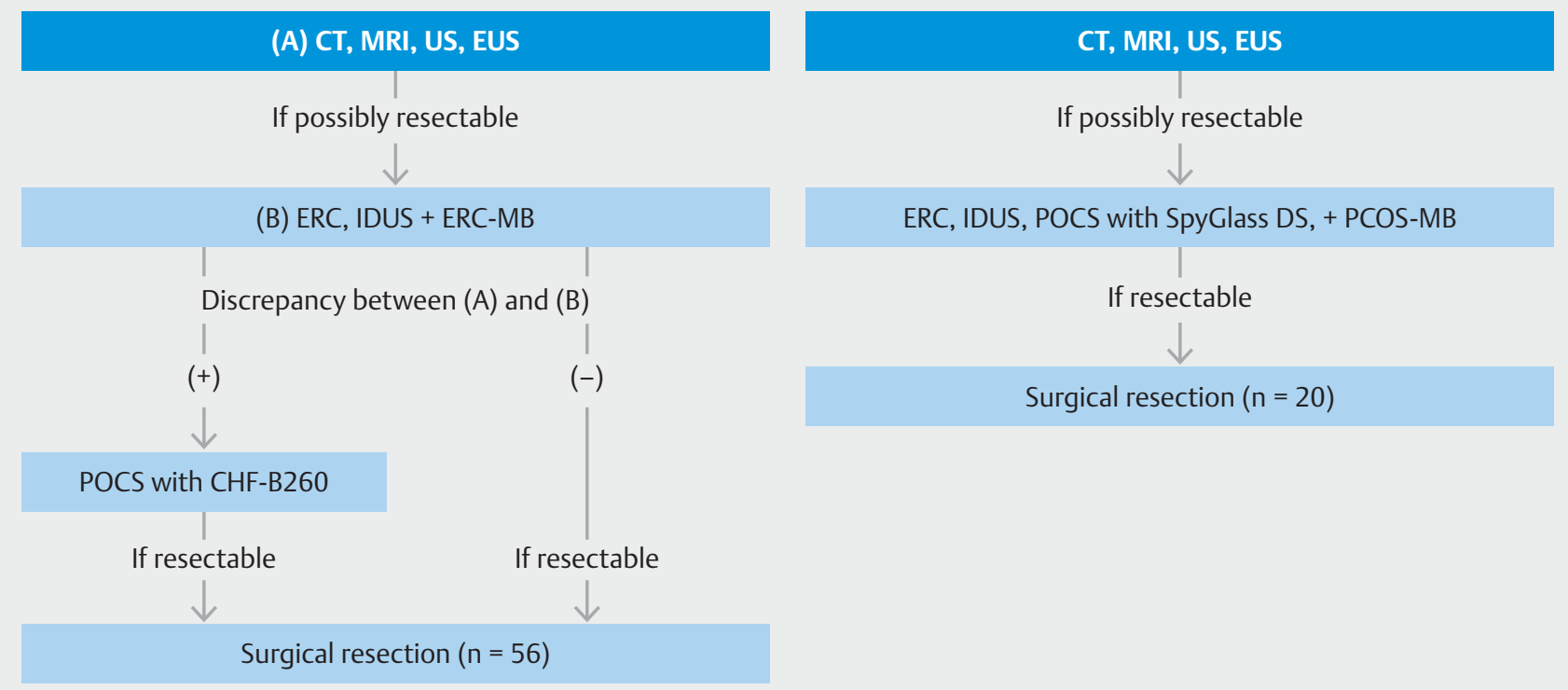

Fig. 2 Diagnostic strategy during each study period. a In group A, fluoroscopy-guided mapping biopsy (ERC-MB) was performed in all patients. Peroral cholangioscopy (POCS) was performed only when the diagnosis of the tumor spread by ERC-MB findings was questionable because of possible discrepancy among examinations. $\mathbf{b}$ In group B, POCS and POCS-guided mapping biopsy (POCS-MB) was performed without reference of ERC-MB in all patients. CT, computed tomography; MRI, magnetic resonance imaging; US, ultrasonography; EUS, endoscopic ultrasonography; ERC, endoscopic retrograde cholangiography; IDUS, intraductal ultrasonography

findings was included as a secondary outcome. The overall preoperative diagnosis was determined in the institutional preoperative conference composed of more than 10 surgeons wherein pancreatobiliary expert endoscopists reviewed all diagnostic information.

Secondary outcome measures were the accuracy of ERCguided mapping biopsy in group A, the ability of POCS to diagnose the borderline(s) of the cancer in the two groups, and the reasons for wrong diagnosis. Borderlines at the liver or ampullary side were not evaluated if the evaluation was deemed unnecessary due to predetermined resection by the required surgical procedure. Additionally, borderlines at the liver and ampullary sides may vary in difficulty. Therefore, results for the liver side and those for the ampullary side were evaluated separately in this study.

Accuracy was defined as precise diagnosis of benignancy or malignancy at the points of interest. For example, when absence of neoplasm at the left/right bifurcation (first bifurcation) was diagnosed in cases of distal bile duct cancer, biopsy results or POCS findings were accurate if the examination confirmed absence of a tumor at that site. When a tumor spread beyond the left/right bifurcation, tumor involvement at the second bifurcation should be estimated for determining the resection line. In such cases, accuracy of the examinations was judged at the second bifurcation, rather than the first. In cases of perihilar cancer that necessitate the decision for pancreatoduodenectomy, the point of interest was determined to be the distal bile duct at the level of the upper margin of the pancreas. Accuracy was not defined as precise diagnosis of the length of the lateral spread or location of the borderline between the neoplasm and non-neoplastic mucosa.

Extent of intraepithelial spread of the neoplasm was judged by referring to the following mucosal findings continuous from the main tumor: (1) fine irregular papillary or granular changes ( Fig.3); (2) fine protrusions with so-called fish egg-like ap-

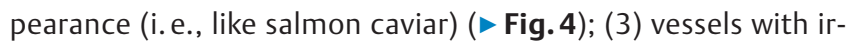
regularity in diameter ( $\triangleright$ Fig.5); and (4) a line demarcating the height of the mucosa ( $\triangleright$ Fig. 6 ).

Resected specimens were evaluated by total segmentation and the extent of the cancer was mapped on a macroscopic picture with segmenting lines. Then, preoperative diagnosis by mapping biopsy plus POCS observation was evaluated as "accurately diagnosed," "underdiagnosed” (false negative), or "overdiagnosed” (false positive).

Sensitivity, specificity, and accuracy were compared between group A and group B.

\section{Analytic methods}

Categorical data were compared by Fisher's exact test. Continual data were compared by $t$ tests. A $P$ value $<0.05$ was considered statistically significant. SPSS software ver. 24 (IBM Japan, Tokyo, Japan) was used for all analyses.

\section{Ethics}

Written informed consent for endoscopic procedures was obtained before the procedure from each patient. This retrospective study was approved by the institutional review board of Sendai City Medical Center. The registration ID issued by UMIN 


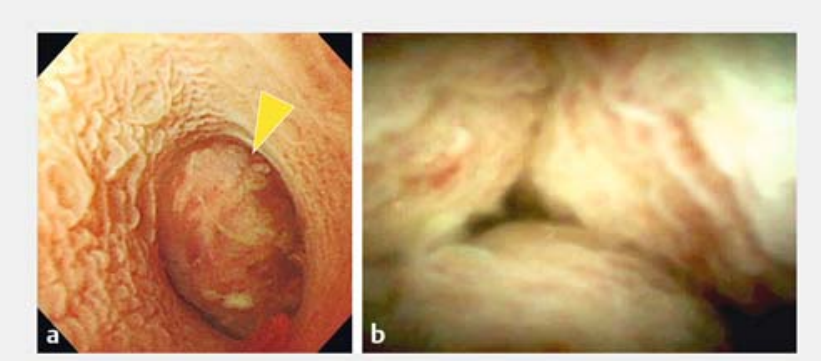

- Fig. 3 Endoscopic images indicating fine irregular or granular changes. a Image obtained by CHF-B260. b Image obtained by SpyGlass DS. Arrow head, main tumor.

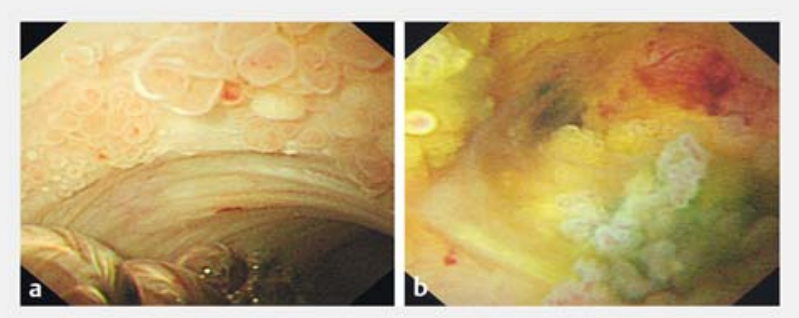

- Fig. 4 Endoscopic images indicating fine protrusions with socalled fish egg-like appearance. Both images are obtained by CHFB260.
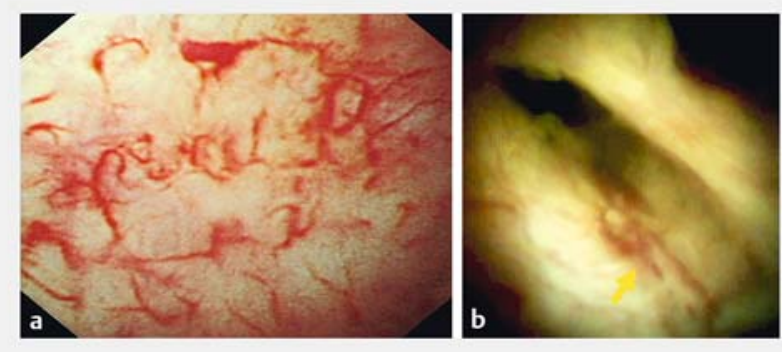

- Fig. 5 Endoscopic images indicating vessels with an irregular diameter. a Image obtained by CHF-B260. b Image obtained by SpyGlass DS (arrow shows an irregular vessel).
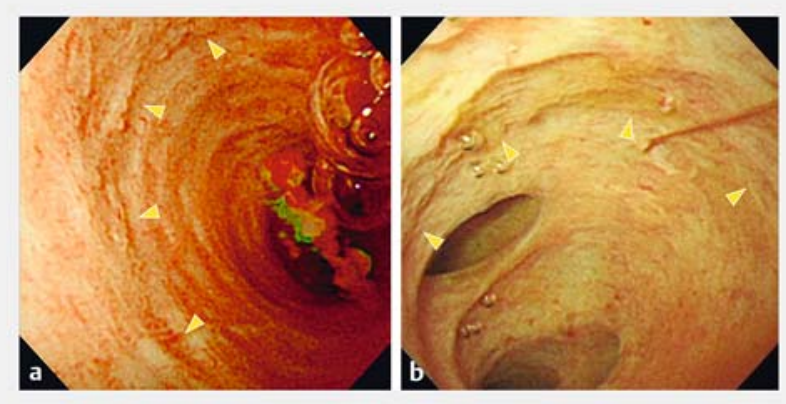

- Fig. 6 Endoscopic images indicating a demarcation line (arrow heads). Both images are obtained by CHF-B260. was UMIN000030583. All authors had access to the study data and reviewed and approved the final manuscript.

\section{Results}

Among 109 patients who underwent surgical resection for extrahepatic bile duct cancer between January 2004 and December 2017, 56 patients for group A and 20 patients for group B were eligible to be included in this study. No statistical differences between the study groups were identified for any baseline characteristics ( $\triangleright$ Table 1 ). Thirty-two patients in group A underwent POCS because of possible discrepancy between ERCguided mapping biopsy and other examinations ( $\triangleright$ Fig. 2 ). The cholangioscope could be advanced beyond the tumor in all patients who required the procedure.

Final accuracy by overall preoperative examinations was $93 \%$ for the liver side and $100 \%$ for the ampullary side in group A, and $84 \%$ for the liver side and $100 \%$ for the ampullary side in group B ( $>$ Table 2 ). Of the four wrongly diagnosed patients in group A, one was overdiagnosed by POCS because of fine granular changes derived from inflammatory hyperplasia after the biopsy showed false-positive results due to contamination; one was overdiagnosed by POCS because of inflammatory changes despite the true negative biopsy results; one was underdiagnosed by POCS despite the true-positive biopsy results, which was wrongly considered to be contamination; and one in whom POCS was not performed was underdiagnosed by the biopsy findings because of sampling errors. The three wrongly diagnosed patients in group B were underdiagnosed by falsenegative results on both POCS and biopsy. Although these three patients had long lateral extent toward the liver side, POCS could not identify mucosal irregularity because the laterally spreading neoplasm resulted in extremely low changes in height. All biopsy specimens obtained under POCS guidance (6, 7, and 8 specimens for the three patients, respectively) were inappropriate because they were too small or did not contain the epithelium. Overall, sensitivity and specificity for liverside estimation were $92 \%$ and $93 \%$, respectively, in group A, and $70 \%$ and $100 \%$, respectively, in group B. Sensitivity and specificity for ampullary-side estimation were $100 \%$ in both groups. No statistical differences in sensitivity or specificity were detected between groups.

Accuracy of ERC-guided mapping biopsy in group A was $80 \%$ $(44 / 51)$ for the liver side and $92 \%$ (12/13) for the ampullary side. Tumor spread toward the liver side was misdiagnosed in seven patients due to contamination in five patients (false-positive), inappropriate biopsy site (i.e., unintended upstream bifurcation of which the tumor spread did not reach) in one patient (false-negative), and impossibility of advancing the biopsy forceps beyond the obstruction in one patient (specimen was not obtained). The biopsy results were false-positive in one patient in whom tumor spread toward the ampullary side was misdiagnosed.

- Table 3 shows the accuracy of simple optical evaluation by POCS for diagnosing borderline(s) of cancer in the two groups. When comparing groups, accuracy rates seemed lower in group B, although statistical significance was not detected. 
- Table 1 Baseline characteristics and lesion description of the patient groups.

\begin{tabular}{|c|c|c|c|}
\hline & $\begin{array}{l}\text { Group A } \\
\mathrm{n}=56\end{array}$ & $\begin{array}{l}\text { Group B } \\
n=20\end{array}$ & $P$ \\
\hline Gender, Male:female & $37: 19$ & $17: 3$ & 0.15 \\
\hline Age, yrs, mean \pm SD & $69 \pm 8$ & $72 \pm 7$ & 0.14 \\
\hline \multicolumn{3}{|l|}{ Macroscopic form } & n.s. \\
\hline - Papillary & 16 & 8 & \\
\hline - Nodular & 35 & 11 & \\
\hline - Flat & 5 & 1 & \\
\hline \multicolumn{3}{|c|}{ Location of the main tumor } & 1.00 \\
\hline - Perihilum & 11 & 4 & \\
\hline - Distal bile duct & 45 & 16 & \\
\hline \multicolumn{4}{|c|}{ Intraepithelial spread (> 10 mm) } \\
\hline " Liver side & 25 & 13 & 0.19 \\
\hline - Ampullary side & 15 & 10 & 0.09 \\
\hline
\end{tabular}

- Table 2 Diagnostic accuracy of overall preoperative examinations.

\begin{tabular}{|l|l|l|l|}
\hline & $\begin{array}{l}\text { Group A } \\
\mathbf{n = 5 6}\end{array}$ & $\begin{array}{l}\text { Group B } \\
\mathbf{n = 2 0}\end{array}$ & $\mathbf{P}$ \\
\hline Liver side & & & \\
\hline - Sensitivity & $92 \%(23 / 25)$ & $70 \%(7 / 10)$ & 0.13 \\
\hline - Specificity & $93 \%(26 / 28)$ & $100 \%(12 / 12)$ & 1.00 \\
\hline - Accuracy & $93 \%(49 / 53)$ & $84 \%(16 / 19)$ & 0.37 \\
\hline Ampullary side & & & \\
\hline - Sensitivity & $100 \%(6 / 6)$ & $100 \%(2 / 2)$ & N/A \\
\hline - Specificity & $100 \%(8 / 8)$ & $100 \%(6 / 6)$ & N/A \\
\hline - Accuracy & $100 \%(14 / 14)$ & $100 \%(4 / 4)$ & N/A \\
\hline
\end{tabular}

\section{Discussion}

It is difficult to accurately diagnose lateral intraepithelial spread of neoplasms of the bile duct [1,2]. Cross-sectional examinations, including CT and MRI, are insufficient to confirm such diagnosis due to the lower resolution of the imaging modalities. Although ultrasonography-based evaluation with EUS and IDUS has been reported to be relatively useful $[6,7]$, mucosal changes must be more precisely estimated by endoscopic optical observation, similar to the estimation of gastrointestinal diseases [21].

The SpyGlass DS system became commercially available on February 2015 in the United States (Boston Scientific Corporation) and on October 2015 in Japan (Boston Scientific Japan K. K., Tokyo, Japan). This system provides digital image processing with a 120-degree field of view, and spectacularly improved im-
- Table 3 Diagnostic accuracy of optical evaluation by POCS.

\begin{tabular}{|c|c|c|c|}
\hline & $\begin{array}{l}\text { Group A } \\
\mathrm{n}=32\end{array}$ & $\begin{array}{l}\text { Group B } \\
n=20\end{array}$ & $P$ \\
\hline \multicolumn{4}{|l|}{ Liver side } \\
\hline - Sensitivity & $88 \%(15 / 17)^{1}$ & $58 \%(7 / 12)$ & 0.09 \\
\hline - Specificity & $83 \%(10 / 12)^{1}$ & $86 \%(6 / 7)$ & 1.00 \\
\hline - Accuracy & $83 \%(25 / 30)^{1}$ & $68 \%(13 / 19)$ & 0.29 \\
\hline \multicolumn{4}{|l|}{ Ampullary side } \\
\hline - Sensitivity & $100 \%(5 / 5)$ & $100 \%(2 / 2)^{1}$ & $\mathrm{~N} / \mathrm{A}$ \\
\hline - Specificity & $100 \%(7 / 7)$ & $100 \%(5 / 5)^{1}$ & $\mathrm{~N} / \mathrm{A}$ \\
\hline - Accuracy & $100 \%(12 / 12)$ & $88 \%(7 / 8)^{1}$ & 0.40 \\
\hline \multicolumn{4}{|c|}{$\begin{array}{l}\text { POCS, peroral cholangioscopy system } \\
{ }^{1} \text { In one case for liver-side evaluation in group A and one case for ampullary- } \\
\text { side evaluation in group B, POCS observation could not be performed for } \\
\text { technical reasons. These cases were included into the accuracy calculation, } \\
\text { but excluded from the sensitivity and specificity calculation. }\end{array}$} \\
\hline
\end{tabular}

age quality in comparison with the previous system from this corporation [22]. Moreover, this system has a dedicated channel for water irrigation, which enables unimpeded procedures using a device such as biopsy forceps or an electronic hydraulic lithotripsy probe without interruption for cleaning. However, this system was considered to be possibly inappropriate for image diagnosis by endoscopic view because of its relatively low image quality in comparison with CHF-B260. Operability was the advantage in the Boston Scientific system, while the image quality was more notable in the Olympus scope.

The results of this study indicate acceptable capability of the SpyGlass DS system for endoscopic diagnosis, including POCSguided biopsy, of the lateral spread. There is similar diagnostic accuracy despite the different image quality due to the high manipulability of the SpyGlass DS scope, which enables greater operator control in visualization, with a four-way tip deflection (the CHF-B260 scope is equipped with a two-way tip deflection). Moreover, the SpyGlass DS scope has a tapered tip that enables easy insertion into the liver side through not only the papilla but also the stricture, resulting in favorable diagnostic outcomes.

In contrast, the capability of optical diagnosis by POCS observation was lower with SpyGlass DS than with CHF-B260 (accuracy rate: $69 \%$ vs. $81 \%$ for the liver side, $80 \%$ vs. $100 \%$ for the ampullary side), which might be due to the lower image quality of the SpyGlass system. Low-growing neoplastic change would be extremely difficult to identify with SpyGlass DS image resolution.

In this feasibility study with a small sample size, overall diagnostic accuracy did not significantly differ between groups A and B. Although the SpyGlass DS system would not be sufficient when only observation is performed, an acceptable strategy involves the addition of POCS-guided biopsy. The SpyGlass DS system could be considered a perfect sheath, which enables exact biopsies with small concern for contamination. 
However, the SpyBite biopsy forceps would not be sufficient to obtain adequate specimens. These forceps appear well-developed with better capability to advance through an extremely thin working channel and superior ability to cut bile duct tissues in comparison with previous ultrathin forceps from other companies for through-the-POCS procedures. However, there were two cases in which all obtained specimens were too small to evaluate. Further improvement in these forceps is desirable.

The small sample size in the retrospective single-center setting is a limitation to this study. The findings may be subject to Type II errors, which risk under-detection of differences in small sample sizes. In studies with a larger number of samples, differences in diagnostic outcomes might be detected. Although the current study of 20 samples per group is able to detect a $38 \%$ difference between two groups with $80 \%$ power with a two-sided significance level of 0.05 by using the Fisher's exact test, 274 samples are required for each group to detect a $10 \%$ difference on equal terms. Moreover, definitive POCS findings for lateral tumor spread have not been fully established, which means that results might differ depending on the endoscopist or observer. However, the impact of such inter-observer disparity might be diminished because the final diagnosis based upon POCS and all other examinations was arrived at after sufficient discussion during the institutional preoperative conference, which was composed of pancreatobiliary experts in this study.

\section{Conclusion}

In conclusion, the SpyGlass DS system was found to be acceptable for diagnosis of the lateral extent of extrahepatic cholangiocarcinoma when cholangioscopy-guided biopsy was applied. Although further improvement in image quality and development of reliable biopsy forceps are desirable, this system could be a standard approach to determine resection lines in an era in which preoperative histological confirmation is increasingly demanded.

\section{Competing interests}

None

\section{References}

[1] Jarnagin WR, Fong Y, DeMatteo RP et al. Staging, resectability, and outcome in 225 patients with hilar cholangiocarcinoma. Ann Surg 2001; 234: $507-517$

[2] Koerkamp BG, Wiggers JK, Allen PJ et al. American Joint Committee on Cancer staging for resected perihilar cholangiocarcinoma: a comparison of the 6th and 7th editions. HPB 2014; 16: 1074- 1082

[3] Wakai T, Shirai Y, Moroda T et al. Impact of ductal resection margin status on long-term survival in patients undergoing resection for extrahepatic cholangiocarcinoma. Cancer 2005; 103: 1210-1216

[4] Igami T, Nagino M, Oda K et al. Clinicopathological study of cholangiocarcinoma with superficial spread. Ann Surg 2009; 249: 296-302
[5] Unno M, Okumoto T, Katayose Y et al. Preoperative assessment of hilar cholangiocarcinoma by multidetector row computed tomography. J Hepatobiliary Pancreat Surg 2007; 14: 434-440

[6] Noda Y, Fujita N, Kobayashi G et al. Intraductal ultrasonography before biliary drainage and transpapillary biopsy in assessment of the longitudinal extent of bile duct cancer. Dig Endosc 2008; 30: 73 - 78

[7] Tamada K, Ido K, Ueno N et al. Preoperative staging of extrahepatic bile duct cancer with intraductal ultrasonography. Am J Gastroenterol 1995; 90: 239-246

[8] Ogawa T, Horaguchi ], Noda Y et al. A case of distal bile duct cancer with extensive intraepithelial spread diagnosed preoperatively by peroral cholangioscopy combined with narrow band imaging. Nihon Shokakibyo Gakkai Zasshi 2010; 107: 112-119

[9] Kawakami H, Kuwatani M, Etoh K et al. Endoscopic retrograde cholangiography versus peroral cholangioscopy to evaluate intraepithelial tumor spread in biliary cancer. Endoscopy 2009; 41: 959-64

[10] Itoi T, Osanai M, Igarashi Y et al. Diagnostic peroral cideo cholangioscopy is an accurate diagnostic tool for patients with bile duct lesions. Clin Gastroenterol Hepatol 2010; 8: 934-938

[11] Nimura Y, Shionoya S, Hayakawa $N$ et al. Value of percutaneous transhepatic cholangioscopy (PTCS). Surg Endosc 1988; 2: 213-219

[12] Yasuda K, Nakajima M, Cho E et al. Comparison of peroral and percutaneous cholangioscopy. Endoscopy 1989; 21: $347-350$

[13] Sato M, Inoue H, Ogawa $S$ et al. Limitations of percutaneous transhepatic cholangioscopy for the diagnosis of the intramural extension of bile duct carcinoma. Endoscopy 1998; 30: 281-288

[14] Tamada K, Kurihara K, Tomiyama T et al. How many biopsies should be performed during percutaneous transhepatic cholangioscopy to diagnose biliary tract cancer? Gastrointest Endosc 1999; 50: 653 658

[15] Lee SS, Kim MH, Lee SK et al. MR cholangiography versus cholangioscopy for evaluation of longitudinal extension of hilar cholangiocarcinoma. Gastrointest Endosc 2002; 56: 25-32

[16] Osanai M, Itoi T, Igarashi Y et al. Peroral video cholangioscopy to evaluate indeterminate bile duct lesions and preoperative mucosal cancerous extension: a prospective multicancer study. Endoscopy 2013; 45: 635-642

[17] Navaneethan U, Hasan MK, Kommaraju K et al. Digital, single-operator cholangiopancreatoscopy in the diagnosis and management of pancreatobiliary disorders: a multicenter clinical experience (with video). Gastrointest Endosc 2016; 84: 649-655

[18] Varadarajulu S, Bang JY, Hasan MK et al. Improving the diagnostic yield of single-operator cholangioscopy-guided biopsy of indeterminate biliary strictures: ROSE to the rescue? (with video) Gastrointest. Endosc 2016; 84: $681-687$

[19] Tanaka R, Itoi T, Honjo M et al. New digital cholangiopancreatoscopy for diagnosis and therapy of pancreaticobiliary deseases (with videos). J Hepatobiliary Pancreat Sci 2016; 23: 220-226

[20] Imanishi M, Ogura T, Kurisu Y et al. A feasibility study of digital singleoperator cholangioscopy for diagnostic and therapeutic procedure (with video). Medicine 2017; 96: e6619

[21] Ogawa T, Ito K, Koshita S et al. Usefulness of cholangioscopic-guided mapping biopsy using SpyGlass DS for preoperative evaluation of extrahepatic cholangiocarcinoma: a pilot study. Endosc Int Open 2018; 06: E204-E204

[22] Laleman W, Verraes K, Van Steenbergen W et al. Usefulness of the single-operator cholangioscopy system SpyGlass in biliary disease: a single-center prospective cohort study and aggregated review. Surg Endosc 2017; 31: $2223-2232$ 